\title{
Choice of pelvic organ prolapse surgery: vaginal or abdominal, native tissue or synthetic grafts, open abdominal versus laparoscopic or robotic
}

\author{
Peter L. Dwyer
}

Published online: 15 July 2014

(C) The International Urogynecological Association 2014

Women with symptomatic pelvic organ prolapse (POP) and their doctors have an increasing number of options and surgical choices. Conservative treatment with a vaginal pessary will successively reduce POP and treat symptoms in most cases, but the patient will require lifetime care of the pessary including regular cleaning and replacement. Surgery is an effective treatment but has operative morbidity and a recurrence rate. The risks of surgery will vary with the type of operation performed. In theory at least, operation selection should be based on what suits the needs of the patient best: what is the most effective and safest way to surgically correct her POP? This is not a simple matter, as what may be more effective may have a higher morbidity.

So it is the doctor's responsibility to explain the operation and its benefits and risks to the patient, and then decide together what operation is to be performed.

In the first instance the decision whether to operate or not should be based on the patient's symptoms, degree of bother, and her medical health. Symptoms should correlate with the examination findings of the site of prolapse and its severity; for instance, women with difficult defecation may have a large rectocele as the cause. Women with chronic pelvic pain will often complain of a vaginal dragging sensation and on examination show mild to moderate prolapse. Is the prolapse causing these symptoms? POP surgery using synthetic mesh, or not, can aggravate the pain. A trial of a vaginal pessary in these women is worthwhile to determine whether relief of POP leads to symptom resolution. Women with chronic pain often tolerate a pessary poorly, and their pain is worse after surgery.

P. L. Dwyer $(\bowtie)$

Department of Urogynecology, Mercy Hospital for Women,

University of Melbourne, Melbourne, Australia

e-mail: pdwyer@connexus.net.au
Once the decision for surgery is made, there are then many choices available to patients and their doctors. In the era of Doctor Google, most patients will be aware of the confusing range of surgical options: vaginal or abdominal, native tissue or synthetic grafts, or open abdominal versus laparoscopic or robotic. They will also be aware of potential risks and their medicolegal sequelae.

Firstly the type of surgery needed will depend on the site of the prolapse. In women with DeLancey level 2 and 3 defects who have symptomatic cystoceles, rectoceles, and perineal defects where apical support is good, most gynaecologists would perform surgery through the vaginal route. For women with marked apical loss of support with uterocervical or vaginal vault prolapse, which is frequently associated with cystocele and enterocele, the options then are whether to repair this vaginally or abdominally, and if abdominally by an open, laparoscopic or a robotic procedure. If the apical POP surgery is performed vaginally, should the suspension be to the uterosacral ligament, the iliococcygeal fascia, or the sacrospinous ligament?

There are advantages and disadvantages of all techniques, and the decision should be based on the patient's needs. Relevant clinical factors in making this decision are the patient, her age and general health, whether further pregnancies are desired, sexual activity, presence of dyspareunia, and vaginal size. The abdominal approach will be preferable in the presence of other abdominal pathology requiring treatment such as an ovarian cyst or when vaginal capacity is already reduced from previous surgery in a sexually active woman. However, the vaginal approach may be preferable in the presence of severe adhesions, which increase the difficulty and risk of the abdominal approach. The risk of recurrence may influence the decision in favour of the abdominal approach and the use of synthetic mesh. Finally, the surgeon's training and experience will and should have an influence on the surgical choice so that the procedure can be completed 
safely. Surgical skill and expertise is rarely discussed or evaluated in the surgical outcomes in trials but may well be one of the most important factors in operative outcomes [1]. Experienced surgeons who have performed many operations have been demonstrated to have better outcomes and lower morbidity in prolapse as well as other areas of surgery. The issue of surgical training and the number of operations and experience necessary to develop appropriate skills to safely and competently perform laparoscopic abdominocolposacropexy is addressed by Deprest and colleagues [2] in this issue of the IUJ. Surgical procedures will be evaluated primarily on effectiveness and safety, but also on cost aspects including cost of the equipment, duration of surgery and hospitalisation, and the surgical training of clinicians.

Therefore, choice of surgery should depend on evidencebased medicine and not be driven by the commercial interests of individual clinicians, or surgical device or medical technology companies. An example of this, I believe, is the use of the term "minimally invasive" used to describe new surgical techniques. It implies to the patient that the procedure is lesser, quicker, and safer. This term was used frequently with the introduction of vaginal mesh devices, laparoscopic procedures, and, more recently, robotic surgery, which seems inappropriate for surgery that takes much longer to perform or has a higher morbidity.

\section{References}

1. Moen M, Noone M, Vassallo B (2014) Anterior colporrhaphy: why surgeon performance is paramount. Int Urogynecol J 25:857-862. doi: 10.1007/s00192-014-2345-6

2. Deprest J, Krofta L, Van der Aa F, Milani AL, Den Boon J, Claerhout F, Roovers JP (2014) The challenge of implementing laparoscopic sacrocolpopexy. Int Urogynecol J. doi:10.1007/s00192-014-2398-6 